\title{
Hidradenocarcinoma metastásico, descripción de un caso y revisión de la literatura
}

\author{
Título corto: Hidradenocarcinoma metastásico \\ Metastatic hidradenocarcinoma, case description and literature review
}

- Tomás Sánchez ${ }^{1}$

' Instituto Nacional de Cancerología.

\section{Resumen}

El hidradenocarcinoma es una neoplasia maligna poco frecuente. No existen guías de consenso para el manejo local o sistémico de la enfermedad.

Se describe el caso de un hombre de 71 años con una lesión de lenta evolución que se llevó a una primera intervención no oncológica con diagnóstico incidental de hidradenocarcinoma de células claras. Posteriormente, se documentó enfermedad metastásica en tórax. Se propuso una primera línea de manejo con capecitabina con progresión luego de cinco ciclos de tratamiento. No se brindaron más líneas por negativa del paciente.

El hidradenocarcinoma se presenta como una lesión nodular asintomática de larga evolución. En la mayoría, el diagnóstico se hace de forma incidental.

El tratamiento incluye la cirugía y la disección ganglionar. La mitad de los pacientes presentan recaída local y, eventualmente, enfermedad metastásica. Los esquemas de manejo de la enfermedad sistémica son variables en composición y resultados.

Palabras clave: neoplasias de las glándulas sudoríparas, metástasis y quimioterapia.

\begin{abstract}
The hidradenocarcinoma is a rare neoplasm. There are no consensus guidelines for the local or systemic treatment of this disease. We describe a case of a 71-year-old male with a slow growing lesion that was treated with a non oncologic resection. The pathologic report informed a clear cell hidradenocarcinoma. After the diagnosis the chest scan documented metastatic disease. It was proposed a first line of chemotherapy using capecitabine, with progression after 5 cycles. The patient refused other chemotherapy lines.

The hidradenocarcinoma usually presents as a long standing asymptomatic nodular lesion in the skin. Most of the diagnoses are made incidentally.

The treatment includes surgery and nodal dissection. Half of the patients present local failure and eventually metastatic disease. The chemotherapy is used with a variety of drugs and results.

Key words: sweat gland neoplasms, metastasis, chemotherapy.
\end{abstract}

\section{Introducción}

Los tumores de glándula sudorípara son neoplasias de presentación poco frecuente y de difícil diagnóstico clínico dado el bajo índice de sospecha, las múltiples entidades relacionadas y la falta de uniformidad de criterios en el diagnóstico diferencial'.

El primer caso de tumor maligno de glándula sudorípara fue publicado por el patólogo francés
Victor André Cornil en 1965². A la fecha son pocos los casos reportados en la literatura con compromiso sistémico y aún no existe un consenso acerca del mejor abordaje diagnóstico, terapéutico o de seguimiento. Se describe a continuación el caso de un paciente con hidradenocarcinoma metastásico que renunció a manejo sistémico luego del fallo de una primera línea.

\section{Recibido el 22 de mayo de 2017; aceptado el 31 de julio de 2017}




\section{Presentación del caso}

Se trata de un varón de 71 años que se presentó con un nódulo a nivel de la piel del muslo izquierdo de más de 20 años de evolución. Fue llevado a resección no oncológica de la lesión y, como hallazgo incidental, se reportó un tumor maligno infiltrante, que se originaba en los anexos de la piel, con positividad para S100, Bcl2, focalmente para ck5/6, AE1AE3, HER2 y receptores hormonales negativos, tiñe compatible con hidradenocarcinoma de células claras. En la revisión de la histología se evidenció compromiso de los bordes se resección, motivo por el cual fue remitido al Instituto Nacional de Cancerología.
Las tomografías de estadificación mostraron un nódulo sólido no calcificado bien definido $(8 \mathrm{~mm})$ en estrecha relación con el aspecto anterior de cisura menor derecha de apariencia inespecífica. No había evidencia de enfermedad en los demás estudios. En evaluación por el grupo quirúrgico se decidió llevar a resección en cuña pulmonar con intención diagnóstica y terapéutica, y, posteriormente, cirugía de ampliación de márgenes y muestreo ganglionar inguinal izquierdo. El análisis histológico de la cuña pulmonar confirmó la presencia de enfermedad metastásica a este nivel, con compromiso de los bordes de resección en la pieza aportada. El espécimen de la ampliación de márgenes
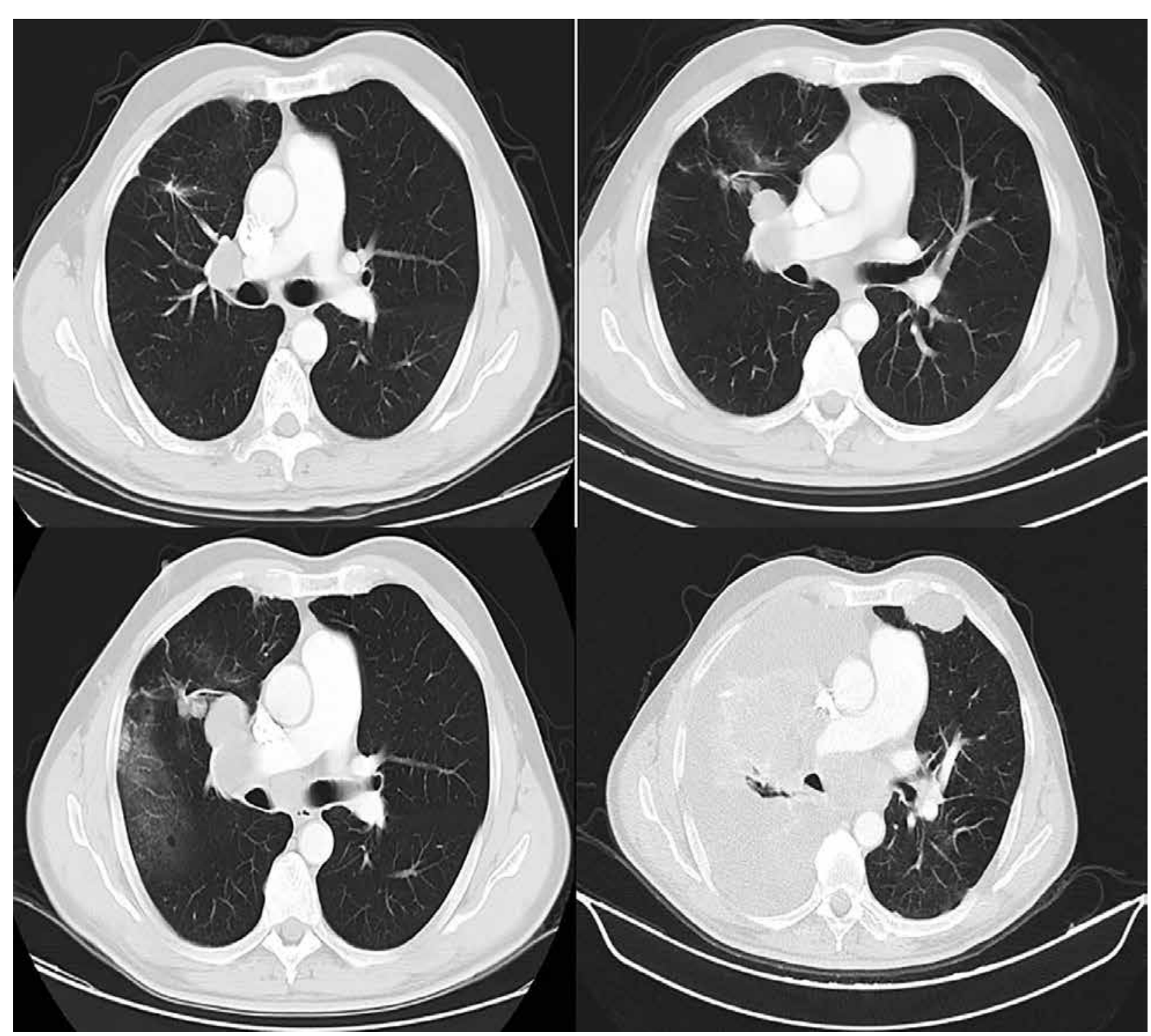

Figura 1. Seguimiento imaginológico por tomografía de las lesiones en tórax. Imagen inicial posterior a la realización de la resección en cuña del pulmón, que muestra una lesión hiperdensa de aproximados $2,5 \mathrm{~cm}$, adyacente al hilio pulmonar derecho (A). Seguimiento a los 4,8 y 12 meses (B, C y D). 
de la piel del muslo y el muestreo ganglionar fueron negativos para tumor maligno.

Luego de la cirugía, fue evaluado por oncología clínica, en donde se decidió hacer seguimiento imaginológico posoperatorio, el cual confirmó la persistencia del compromiso metastásico pulmonar y documentó además un nuevo compromiso mediastinal. El grupo quirúrgico consideró que no era candidato a resección por la ausencia de plano de clivaje con estructuras vasculares del mediastino. Se brindó una primera línea para enfermedad metastásica con capecitabina $2000 \mathrm{mg} / \mathrm{m}^{2} /$ día días 1 a 14. Completó cinco ciclos de dicho esquema; los estudios de revaloración mostraron progresión de las lesiones pulmonares y mediastinales, lo que, en asociación a toxicidad grado 4 a nivel gastrointestinal, motivó la suspensión del esquema.

Se le propuso al paciente una segunda línea para el manejo de su enfermedad con base en carboplatino paclitaxel, sin embargo, este rechazó la propuesta y decidió continuar en seguimiento, dado que a la fecha no presentaba síntoma alguno relacionado con su enfermedad. Se hizo un primer seguimiento a los tres meses de suspendido el tratamiento, con imágenes del tórax que mostraron progresión de su enfermedad a nivel del mediastino y parénquima pulmonar; a pesar de los hallazgos, el paciente no aceptó tratamiento sistémico. En el momento se encuentra en seguimiento imaginológico con evidencia de progresión de su enfermedad; no obstante, rechaza el manejo con quimioterapia (figuras 1 y 2).

\section{Discusión}

Según datos publicados por SEER, los carcinomas apocrinos/ecrinos en sumatoria alcanzan una tasa de incidencia de 5,1 por 1 millón de personas/año, cifra que viene en aumento en las últimas décadas. Se presentan con mayor frecuencia a mayor edad, en especial en los hombres blancos no hispánicos ${ }^{3}$.

El hidradenocarcinoma es el tercero en frecuencia de este grupo, y representa el $8,1 \%$ de todos los carcinomas ecrios/apocrinos, con una incidencia de 0,4 por 1 millón de personas/año ${ }^{4}$.

Similar a la presentación de nuestro paciente, en la literatura se describe el diagnóstico de esta enfermedad como un hallazgo incidental en el análisis histológico de una lesión aparentemente benigna. Usualmente se manifiesta en mayores de 30 años como un nódulo o masa de localización variable (cabeza y cuello), de crecimiento lento y asintomático ${ }^{5}$.

Comparte las características histológicas del hidradenoma, sin embargo, se diferencia por el patrón infiltrante profundo, invasión linfovascular y perineural, necrosis, pleomorfismo nuclear y numerosas mitosis. Es frecuente el compromiso metastásico a distancia, usualmente afectando el pulmón, hueso, cerebro, hígado y otras partes de la piel ${ }^{6,7}$.

Desde el punto de vista inmunofenotípico, estos tumores son positivos para marcadores epiteliales como citoqueratinas, EMA, proteína S100 y ACE. Se ha reportado que un $30 \%$ de los hidradenocarcinomas son positivos para receptores de estrógenos, en menor frecuencia expresan HER2 amplificado (12\% al $36 \%$ dependiendo de las series) y otros tienen fuerte positividad para EGFR en inmunohistoquímica, pero a la fecha no se ha reportado ninguno con amplificación de este receptor. Este hallazgo hace pensar en un mecanismo epigenético como responsable de la expresión del receptor ${ }^{8,9}$.

Desde el punto de vista genotípico, se han descrito mutaciones en TP53 con una prevalencia del $30 \%$ y mutaciones susceptibles de terapias diana, como PTCH1 (inhibidor Shh) y TCF7L1 (inhibidor Wnt $)^{10,11}$.

El manejo de la enfermedad temprana se basa en un esquema de tres pasos que incluye la resección local temprana con márgenes oncológicos, el vaciamiento ganglionar y el seguimiento a largo plazo. No está definido el rol de intervenciones, como el ganglio centinela o la quimioterapia/radioterapia adyuvantes ${ }^{12}$. Se debe tener en cuenta que, a pesar del manejo quirúrgico radical y la terapia adyuvante, la recurrencia local y regional del tumor es la regla, presentándose en el 50\% al $60 \%$ de los $\operatorname{casos}^{13}$.

Para el manejo sistémico de la enfermedad recurrente, irresecable o metastásica, los esquemas se soportan en series de casos. Dentro de los publicados se encuentran: cisplatino/vinorelbine ${ }^{14}$, carboplatino/paclitaxe ${ }^{15}$, cisplatino/doxorrubicina/ mitomicina o cisplatino/vincristina ${ }^{16}, \mathrm{VAC}+$ bleomicina, 5-FU + cisplatino y radioterapia ${ }^{17}$ y terapias monoagente a base de capecitabina o 5-fluoruracilo $^{18,19}$. En conjunto, en los reportes se encuentran resultados variables, que van desde la respuesta completa con esquemas basados en tres agentes, 
y tiempos a la progresión, que van desde 6 hasta 24 meses $^{14-19}$

Se ha descrito, así mismo, manejo con terapias dirigidas, dentro de las cuales se encuentran: tamoxifeno ${ }^{20}$, sunitinib ${ }^{21}$, cisplatino más cetuximab ${ }^{22}$ y trastuzumab ${ }^{23}$. Los resultados son variables con datos de tiempo a la progresión entre los 2 y 36 meses.

Para la paliación de los síntomas locales, la radioterapia se ha usado con dosis que van de los 50 a los 70 Gy. Algunos incluso han sugerido manejo con radioterapia adyuvante en tumores de más de $5 \mathrm{~cm}$, márgenes positivos, alto grado histológico o en presencia de invasión linfovascular ${ }^{24}$. Otros han reportado resultados favorables con electroquimioterapia en tumores de cabeza y cuello 25

El pronóstico de la enfermedad en progresión es malo, con supervivencia reportada a cinco años de tan solo un $30 \%{ }^{13}$. Se han descrito como factores pronósticos la tasa mitótica, el espesor del compromiso y la invasión linfovascular²6.

\section{Conclusión}

El hidradenocarcinoma es una neoplasia poco frecuente y de diagnóstico incidental en la mayoría de los casos. Dentro del grupo de los tumores de glándula sudorípara, este se caracteriza por una alta tasa de recurrencia local y de metástasis a distancia. Se describió el caso de un paciente con diagnóstico incidental de hidradenocarcinoma que debutó con una enfermedad local de evolución muy lenta. Luego del manejo inicial no oncológico, se demostró enfermedad metastásica que progresó a uno de los esquemas que ha demostrado mayor beneficio en la literatura. Este caso nos ratifica la importancia de tener un alto índice de sospecha para el diagnóstico temprano, toda vez que no existen esquemas estándar para el manejo sistémico que hayan demostrado un beneficio contundente en el contexto de la enfermedad metastásica por el hidradenocarcinoma.

\section{Referencias}

1. Cardoso JC, Calonje E. Malignant sweat gland tumours: an update. Histopathology 2015;67(5):589-606.

2. Gates O, Warren S, Warvi WN. Tumors of sweat glands. Am J Pathol 1943;19(4):591-631.

3. Blake PW, Bradford PT, Devesa SS, Toro JR. Cutaneous appendageal carcinoma incidence and survival patterns in the
United States: a population-based study. Arch Dermatol 2010;146(6):625-32.

4. Bhullar JS, Varshney N, Mervak T, Mittal V, Silapaswan S. Hidradenocarcinoma: a sinister diagnosis of a suspected benign condition. Am Surg 2014;80(12):1271-2.

5. Gauerke S, Driscoll JJ. Hidradenocarcinomas: a brief review and future directions. Arch Pathol Lab Med 2010;134(5):781-5.

6. Massad LS, Bitterman P, Clarke-Pearson DL. Metastatic clear cell eccrine hidradenocarcinoma of the vulva: survival after primary surgical resection. Gynecol Oncol 1996;61(2):287-90.

7. Messing MJ, Richardson MS, Smith MT, King L, Gallup DG Metastatic clear-cell hidradenocarcinoma of the vulva. Gynecol Oncol 1993;48(2):264-8

8. Hasebe $T$, Mukai K, Ishihara K, Kaneko A, Shimosato Y. Sebaceous gland and sweat gland carcinomas of the skin. Clinicopathological study and significance of c-erbB-2 oncoprotein expression. Acta Pathol Jpn 1992;42(8):585-94.

9. Piris A, Scopsi L, Clemente C, Cetti Serbelloni F, Mihm MC Jr, Hoang MP. Epidermal growth factor receptor gene status by fluorescence in situ hybridization in malignant, atypical, and benign hidradenomas. Am J Dermatopathol 2010;32(6):586-92.

10. Gupta E, Guthrie KJ, Krishna M, Asmann Y, Parker AS, Joseph RW. Whole exome sequencing of a patient with metastatic hidradenocarcinoma and review of the literature. Rare Tumors 2015;7(1):5719.

11. Takata $M$, Hashimoto $K$, Mehregan $P$, Lee MW, Yamamoto $A$, Mohri $S$, et al. Genetic changes in sweat gland carcinomas. J Cutan Pathol 2000;27(1):30-5

12. Delgado R, Kraus D, Coit DG, Busam KJ. Sentinel lymph node analysis in patients with sweat gland carcinoma. Cancer 2003;97(9):2279-84

13. Elder DE, Elensitas R, Johnson BL, Murphy GF. Elders's histopathology of the skin. Chapter 30 Tumors of the epidermal appendages. 10th ed. Philadelphia, PA: Lippincott Williams \& Wilkins; 2009.

14. Wang $X X$, Wang HY, Zheng JN, Sui JC. Primary cutaneous sweat gland carcinoma. J Cancer Res Ther 2014;10(2):390-2.

15. Tlemcani K, Levine D, Smith RV, Brandwein-Gensler M, Staffenberg DA, Garg MK, et al. Metastatic apocrine carcinoma of the scalp: prolonged response to systemic chemotherapy. J Clin Oncol 2010;28(24):e412-4.

16. Piedbois P, Breau JL, Morere JF, Israel L. Sweat gland carcinoma with bone and visceral metastases. Prolonged complete remission lasting 16 months as a result of chemotherapy. Cancer 1987;60(2):170-2.

17. Chintamani, Sharma R, Badran R, Singhal V, Saxena S, Bansal A. Metastatic sweat gland adenocarcinoma: a clinico-pathological dilemma. World J Surg Oncol 2003;1(1):13.

18. Lerner A, Beckford A, Ugent S, Goldberg L, Jalisi S, Demierre M. Complete response of metastatic malignant hidradenocarcinoma to capecitabine treatment. Arch Dermatol 2011;147(8):998-9.

19. Jouary $T$, Kaiafa $A$, Lipinski $P$, Vergier $B$, Lepreux $S$, Delaunay $M$, et al. Metastatic hidradenocarcinoma: efficacy of capecitabine. Arch Dermatol 2006;142(10):1366-7.

20. Sridhar KS, Benedetto P, Otrakji CL, Charyulu KK. Response of eccrine adenocarcinoma to tamoxifen. Cancer 1989;64(2):366-70.

21. Battistella M, Mateus $C$, Lassau N, Chami L, Boukoucha M, Du- 
villard $P$, et al. Sunitinib efficacy in the treatment of metastatic skin adnexal carcinomas: report of two patients with hidradenocarcinoma and trichoblastic carcinoma. J Eur Acad Dermatol Venereol 2010;24(2):199-203.

22. Gallerani E, Ciriolo M, Rossini C, Cavalli F. Axillary apocrine carcinoma with brain metastases. J Clin Oncol 2007;25(35):5655-6.

23. Nash JW, Barrett TL, Kies M, Ross MI, Sneige N, Diwan AH, et al. Metastatic hidradenocarcinoma with demonstration of Her-2/ neu gene amplification by fluorescence in situ hybridization: potential treatment implications. J Cutan Pathol 2007;34(1):49-54.
24. Chamberlain RS, Huber K, White JC, Travaglino-Parda R. Apocrine gland carcinoma of the axilla: review of the literature and recommendations for treatment. Am J Clin Oncol 1999;22(2):131-5.

25. Kyrgias G, Kostopoulou E, Zafiriou E, Zygogianni A, Skarlatos J, Roussaki-Schulze AV, et al. Hidradenocarcinoma of the temporal area successfully treated with concomitant electrochemotherapy and radiotherapy. Head Neck Oncol 2013;5(2):14.

26. Soni A, Bansal N, Kaushal V, Chauhan AK. Current management approach to hidradenocarcinoma: a comprehensive review of the literature. Ecancermedicalscience 2015;9:517. 